\title{
Apremilast prevents IL-17-induced cellular senescence in ATDC5 chondrocytes mediated by SIRT1
}

\author{
BENLONG WANG, WEI SUN, KUNWEI BI, YONG LI and FENG LI \\ Department of Sports Medicine of Zaozhuang Municipal Hospital, Zaozhuang, Shandong 277000, P.R. China
}

Received August 17, 2020; Accepted November 30, 2020

DOI: $10.3892 / \mathrm{ijmm} .2021 .4845$

\begin{abstract}
Osteoarthritis is the most prevalent joint degenerative disease and has been considered a major cause of severe joint pain and physical disability in the elderly. The chondrocyte is the only cell type found in articular cartilage and chondrocyte senescence plays a pivotal role in the pathogenesis of osteoarthritis. Apremilast is an oral PDE4 inhibitor and has been used for the treatment of patients with active psoriatic arthritis. In the present study, the biological function of apremilast was examined in an interleukin (IL)-17-treated chondrocyte model. Expression levels of target genes and proteins were measured using reverse transcription-quantitative PCR, ELISA, and western blotting, respectively. ROS levels in chondrocytes were examined using the fluorescent dye DCFH-DA. Cellular senescence was determined using senescence-associated- $\beta$-galactosidase staining. The profile of cell cycle phases was analyzed via flow cytometry. It was revealed that treatment with apremilast reduced the expression of IL- $1 \beta, \mathrm{MCP}-1$, and the production of ROS. SA- $\beta$-gal staining results indicated that the presence of apremilast suppressed IL-17-induced cellular senescence. Furthermore, apremilast prevented IL-17-induced G0/G1 phase cell cycle arrest. In addition, it was demonstrated that apremilast suppressed IL-17-induced expression of p21 and PAI-1. Notably, the silencing of sirtuin 1 (SIRT1) abolished the protective effect of apremilast against IL-17-induced cellular senescence, suggesting that the action of apremilast in chondrocytes is dependent on SIRT1. In conclusion, the present results revealed that apremilast exerted a beneficial effect, thereby protecting chondrocytes from senescence induced by IL-17.
\end{abstract}

Correspondence to: Dr Feng Li, Department of Sports Medicine of Zaozhuang Municipal Hospital, 41 Longtou Road, Shizhong, Zaozhuang, Shandong 277000, P.R. China

E-mail: lifeng245@163.com

Key words: osteoarthritis, chondrocyte senescence, apremilast, plasminogen activator inhibitor-1, sirtuin 1

\section{Introduction}

Osteoarthritis (OA), is a painful degenerative joint disease that occurs in the elderly across the developed and developing world and has attracted increasing attention in the past decades (1). Although the pathological mechanism of the disease is complicated, aging is considered as the main risk factor in the development of OA (2). The chondrocyte is the only cell type found in articular cartilage. Healthy chondrocytes are responsible for maintaining cartilage homeostasis (3). However, in the condition of OA, chondrocytes produce various pro-inflammatory cytokines and chemokines, which lead to chronic inflammation. Furthermore, high levels of these cytokines result in chondrocyte senescence (4). Cellular senescence plays an important role in $\mathrm{OA}$ through $\mathrm{G} 0 / \mathrm{G} 1$ phase cell cycle arrest and limiting the proliferative and synthesizing capacities of chondrocytes, resulting in disability of chondrocytes to secrete extracellular matrix components (2). Several signaling pathways have been implicated in the process of cellular senescence. For example, increased expression of plasminogen activator inhibitor-1 (PAI-1) and p21 have been considered as important biomarkers of cellular senescence. Additionally, sirtuin 1 (SIRT1), a nicotinamide dinucleotide $\left(\mathrm{NAD}^{+}\right)$-dependent deacylase, plays diverse roles in negatively regulating senescence, aging, and inflammation in different types of cells, including chondrocytes (5). The ATDC5 chondrocyte cell line.

Interleukin (IL)-17 is well known as a member of a multifunctional cytokine family. Research has demonstrated that IL-17 plays an important role in several inflammatory diseases, such as psoriasis, multiple sclerosis (MS), and rheumatoid arthritis (6). Furthermore, studies have revealed the positive relationship between IL-17 concentration and the severity of OA $(7,8)$. The antibody fragment of IL-17 has been used for the treatment of rheumatoid arthritis (9). Although the successful application of IL-17 inhibitors in OA patients has not been reported to date, efforts have been made by both clinical physicians and scientific pharmacologists (10). IL-17 could induce the production of pro-inflammatory cytokines by chondrocytes, thereby triggering a sustained inflammatory response (11). Furthermore, IL-17 enhances the release of matrix metalloproteinases (MMPs), which lead to joint damage and cartilage destruction (12). However, the biological function of IL-17 in chondrocytes remains to be elucidated. 
Apremilast, an oral phosphodiesterase 4 (PDE4) inhibitor, was approved by the US Food and Drug Administration (FDA) in 2014 for the treatment of patients with active psoriatic arthritis and plaque psoriasis (13). Evidence has revealed that apremilast could inhibit PDE4 activity and blocks pro-inflammatory cytokines such as tumor necrosis factor (TNF)- $\alpha$, IL-1 $\beta$, IL-17, and IL-2 in the inflammatory response of psoriasis (14). These results indicate that apremilast may have a protective effect on other inflammatory diseases. Notably, the administration of apremilast revealed efficacy in an atopic dermatitis (AD) mouse model by reducing ear swelling and the expression of monocyte chemoattractant protein-1 (MCP-1), IL-12/IL-23p40, and IL-31 (15). Notably, apremilast has been reported to induce an increase of the anti-inflammatory B-cell subset Breg cells, and a decrease of Th1 cells, IFN $\gamma$-producing NKT cells, and IL-17-producing NKT cells (16). However, it is still unknown whether apremilast possesses any efficacy in OA.

\section{Materials and methods}

Cell culture and treatment. The ATDC5 chondrocyte cell line was obtained from the Cell Bank of the Cell Engineering Division, RIKEN BioResource Research Center. The cell line was maintained in DMEM/F12 medium (cat. no. 12634010) and supplied with $10 \%$ FBS (cat. no. 10100147; both from Gibco; Thermo Fisher Scientific, Inc.) in a $5 \% \mathrm{CO}_{2}$ humidified cell culture incubator at $37^{\circ} \mathrm{C}$. For the treatment experiment, the cells were plated in 6 -well cell culture plates and treated with IL-17 (10 ng/ml) (R\&D Systems, Inc.) in the presence or absence of apremilast $(0.5$ and $1 \mu \mathrm{M})$ (MedChemExpress) at $37^{\circ} \mathrm{C}$ for $24 \mathrm{~h}$.

$R N A$ isolation and reverse transcription-quantitative (RT-q) $P C R$. Total RNA was isolated from ATDC5 cells using TRIzol (cat. no. 15596018; Thermo Fisher Scientific, Inc.) following the manufacturer's protocol. The total RNA was treated with DNAase (cat. no. M0303S; New England Biolabs, Inc.) and then quantified using a Nanodrop spectrophotometer (Thermo Fisher Scientific, Inc.). RNA (2 $\mu \mathrm{g})$ was reversely transcribed into cDNA using an NGS Reverse Transcription kit (cat. no. A45003; Thermo Fisher Scientific, Inc.) according to the manufacturer's instructions. Gene expression was quantified using the SYBR Green PCR Master Mix (cat. no. 4309155; Thermo Fisher Scientific, Inc.) on an ABI Prism 7000 PCR system (Applied Biosystems; Thermo Fisher Scientific, Inc.) with the following thermocycling conditions: An initial denaturation and enzyme activation at $95^{\circ} \mathrm{C}$ for $10 \mathrm{~min}$, followed by 35 cycles of $30 \mathrm{sec}$ denaturation at $95^{\circ} \mathrm{C}$, an attachment of primers for $1 \mathrm{~min}$ at $60^{\circ} \mathrm{C}$, and extension at $72^{\circ} \mathrm{C}$ for $30 \mathrm{sec}$, and finally one cycle at $72^{\circ} \mathrm{C}$ for $10 \mathrm{~min}$ for final elongation. The following primers were used in the present study: IL- $1 \beta$ forward, 5'-TGGACCTTCCAGGATGAGGACA-3' and reverse, 5'-GTTCATCTCGGAGCCTGTAGTG-3'; MCP-1 forward, 5'-CCCAGGAGTGCCTTGATTC-3' and reverse, 5'-CGCCCCATAATTCTGACATC-3'; p21 forward, 5'-GTT CATCTCGGAGCCTGTAGTG-3' and reverse, 5'-AGCTGG CCTTAGAGGTGA-3'); PAI1 forward, 5'-GACACCCTC AGCATGTTCATC-3' and reverse, 5'-AGGGTTGCACTA AACATGTCAG-3'; SIRT1 forward, 5'-TTGTGAAGCTGT
TCGTGGAG-3' and reverse, 5'-GGCGTGGAGGTTTTTCA GTA-3'; and GAPDH (forward, 5'-AAGAGGGATGCTGCC CTTAC-3' and reverse, 5'-CCATTTTGTCTACGGGACGA-3'.

The $2^{-\Delta \Delta \mathrm{Cq}}$ method (17) was used to calculate the relative expression of the target genes, and the expression of GAPDH was used as the internal control.

\section{2,7-Dichlorodihydrofluorescein diacetate (DCFH-DA)} staining. Reactive oxygen species (ROS) levels in chondrocytes were measured using the fluorescent dye DCFH-DA (Sigma-Aldrich; Merck KGaA). Briefly, chondrocytes $\left(1 \times 10^{5}\right.$ cells $\left./ \mathrm{ml}\right)$ were treated with IL-17 or apremilast as aforementioned, then incubated with diluted $5 \mu \mathrm{M}$ DCFH-DA in the dark for $60 \mathrm{~min}$ at $37^{\circ} \mathrm{C}$. Then, the cells were washed with PBS and images were captured with a confocal laser-scanning microscope (Leica TCS SL) (magnification, x10) equipped with an argon laser. The fluorescent quantification was analyzed using the software ImageJ (v2.1.4.7; National Institutes of Health).

Senescence-associated- $\beta$-galactosidase (SA- $\beta$-gal) staining. Cellular senescence was determined using senescence-associate d- $\beta$-galactosidase (SA- $\beta$-gal) staining by a Cellular Senescence Assay kit (product code ab228562; Abcam) according to the instructions from the manufacturer. Briefly, the cells were quickly washed with PBS twice and then fixed with $4 \%$ paraformaldehyde (PFA) for $15 \mathrm{~min}$ at room temperature followed by incubation with the staining solution overnight at $37^{\circ} \mathrm{C}$. The number of SA- $\beta$-gal ${ }^{+}$cells were counted from the total number of cells. The images were analyzed using ImageJ (v2.1.4.7).

Protein isolation and western blotting. Total protein from chondrocytes was lysed using RIPA buffer (cat. no. R0010; Solarbio Life Sciences). The protein concentration was measured using the Pierce BCA protein assay kit (cat. no. 23225; Thermo Fisher Scientific, Inc.). A total of $20 \mu \mathrm{g}$ of protein was separated by $8-12 \%$ sodium dodecyl sulfate-polyacrylamide electrophoresis (SDS-PAGE) gel and transferred to polyvinylidene difluoride (PVDF) membranes (EMD Millipore). The blots were then blocked with 5\% slim milk diluted in TBST for $1 \mathrm{~h}$ at room temperature. The blots were then separately incubated with the following primary antibodies: p21 (1:1,000; cat. no. sc-6246; Santa Cruz Biotechnology, Inc.), PAI-1 (1:2,000; cat. no. 66261-1-Ig; ProteinTech Group, Inc.), SIRT1 (1:1,000; product no. 2310; Cell Signaling Technology, Inc.), $\beta$-actin (1:10,000; cat. no. AF7018; Affinity Biosciences) in a cold room $\left(4^{\circ} \mathrm{C}\right)$ overnight, and followed by incubation with HRP-conjugated goat anti-mouse $\operatorname{IgG}(\mathrm{H}+\mathrm{L})$ secondary antibody $(1: 2,000$; cat. no. 31430$)$ or goat anti-rabbit $\operatorname{IgG}(\mathrm{H}+\mathrm{L})$ secondary antibody (1:2,000; cat. no. G-21234; both from Thermo Fisher Scientific, Inc.) for $1 \mathrm{~h}$ at room temperature. Immunoblots were detected with Amersham ${ }^{\mathrm{TM}}$ ECL $^{\mathrm{TM}}$ Prime reagent (GE Healthcare Life Sciences; Cytiva). The images were captured and analyzed with an EC3 UVP imaging system (Cole-Parmer). The densitometry data were analyzed using the software Image J (v2.1.4.7).

ELISA. The mouse MCP-1 ELISA kit (cat. no. SEKM-0108) and mouse IL-1 $\beta$ ELISA kit (cat. no. SEKM-0002) were purchased from Sobarbio Life Sciences. The secretions of 
IL-1 $\beta$ and MCP-1 in chondrocytes were determined according to the manufacturer's instructions. The optical density of the reaction was detected using a 96-well plate reader (BioTek Instruments, Inc.).

Cell cycle analysis. The cell cycle of ATDC5 chondrocytes was assessed with flow cytometry. After IL-17 or apremilast treatment as aforementioned, $1.0 \times 10^{6}$ cells were collected and fixed with $70 \%$ ethanol at $4^{\circ} \mathrm{C}$ overnight. Cells were then permeabilized with $0.1 \%$ Triton X-100 in Tween-20, followed by staining using propidium iodide (PI) $(50 \mathrm{mg} / \mathrm{l})$ (Sigma-Aldrich, USA) on ice for $5 \mathrm{~min}$. The profile of cell cycle phases was analyzed via flow cytometry on the BD LSRII system (BD Biosciences). The results were analyzed with FlowJo analytical software (version X; Tree Star, Inc.).

SIRT1 siRNA transfection. ATDC5 chondrocytes ( $2 \times 10^{5}$ cells/well) were plated in 6-well plates. SIRT1-specific or non-specific siRNA (100 nM; Guangzhou RiboBio Co., Ltd.) in serum-free medium was then transfected into cells using Lipofectamine RNAiMax (Invitrogen; Thermo Fisher Scientific, Inc.). The sequence of SIRT1 siRNA was: Forward, 5'-AGAUAUCAAUACAAUUGA AdTdT-3' and reverse, 5'-UUCAAUUGUAUUGAUAUCUdTdT-3'. After $4 \mathrm{~h}$ of transfection at $37^{\circ} \mathrm{C}$, the medium was replaced with normal medium containing $10 \%$ FBS and incubation was continued for $48 \mathrm{~h}$.

Statistical analysis. Data are presented as the mean \pm SEM. Statistical analysis was performed using SPSS version 24 software (IBM Corp.), and the difference between groups was assessed using ANOVA followed by Bonferroni's test. $\mathrm{P}<0.05$ was considered to indicate a statistically significant difference.

\section{Results}

Apremilast reduces $I L-17$-induced expression levels and secretions of pro-inflammatory cytokines. To explore the protective effects of apremilast (Fig. 1A) in chondrocytes, the effects of apremilast on the production of pro-inflammatory cytokines were assessed. Compared with the control group, IL-17 treatment significantly increased the expression levels and secretions of IL-1 $\beta$ and MCP-1 at both mRNA and protein levels, which were reduced by apremilast in a dose-dependent manner (Fig. 1B and C). This result indicated that apremilast has a potential inhibitory effect on the production of pro-inflammatory cytokines.

Apremilast suppresses IL-17-induced production of ROS. Aberrant ROS production plays a pivotal role in cellular senescence. In the present study, it was examined whether apremilast influenced the generation of ROS. As anticipated, the DCFH-DA staining revealed that IL-17 alone induced $\sim 3.3$-fold ROS production, but the presence of 0.5 and $1 \mu \mathrm{M}$ concentrations of apremilast decreased IL-17-induced ROS production to only 2.4- and 1.7-fold, respectively (Fig. 2).

Apremilast prevents IL-17-induced cellular senescence. Since apremilast revealed its inhibitory effect on the production of pro-inflammatory cytokines and ROS production induced by
A

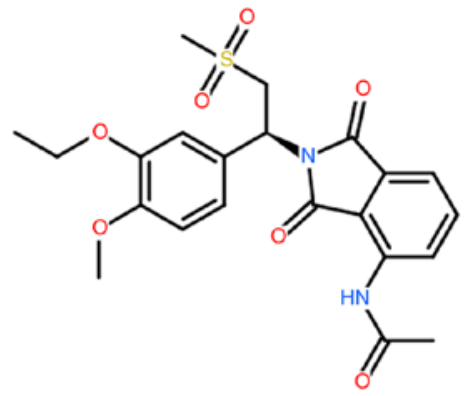

B $\quad$ Control

口 IL-17 (10 ng/ml)

ㅁ IL-17 $(10 \mathrm{ng} / \mathrm{ml})+$ apremilast $0.5 \mu \mathrm{M}$

ㅁ IL-17 $(10 \mathrm{ng} / \mathrm{ml})+$ apremilast $1 \mu \mathrm{M}$
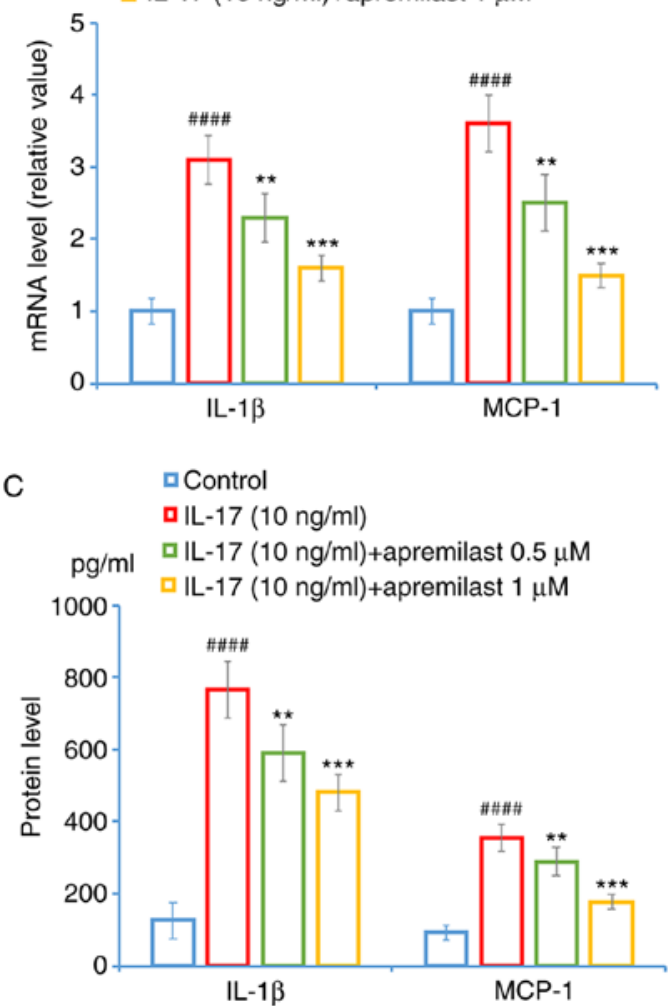

Figure 1. Apremilast prevents IL-17-induced expression and secretions of pro-inflammatory cytokines in mouse ATDC5 chondrocytes. Cells were treated with IL-17 $(10 \mathrm{ng} / \mathrm{ml})$ in the presence or absence of apremilast $(0.5$ and $1 \mu \mathrm{M})$ for $24 \mathrm{~h}$. (A) Molecular structure of apremilast. (B) mRNA levels of IL- $1 \beta$ and MCP-1. (C) Secretions of IL- $1 \beta$ and MCP-1. ${ }^{\# \# \# P} \mathrm{P}<0.0001$ vs. the vehicle control group; ${ }^{* *} \mathrm{P}<0.01$ and ${ }^{* * *} \mathrm{P}<0.001$ vs. the IL-17 group. IL, interleukin; MCP-1, monocyte chemoattractant protein-1.

IL-17, it was predicted that apremilast could protect chondrocytes from senescence. As revealed in Fig. 3, IL-17 stimulation induced a 3.7 -fold increase of SA- $\beta$-gal activity, while $1 \mu \mathrm{M}$ apremilast reduced its activity to only 2.1 -fold.

Apremilast prevents IL-17-induced cell cycle arrest in the G0/G1 phase. Next, the cell cycle profile was assessed with flow cytometry. Compared to non-treated cells, 7 days of IL-17 stimulation induced a 19\% increase in G0/G1 phase cell cycle arrest. In addition, $\mathrm{G} 2 / \mathrm{M}$ and $\mathrm{S}$ phase cells were reduced from 21.8 and 22 to 17.1 and $7.6 \%$, respectively, by the same IL-17 treatment. However, in the presence of $1 \mu \mathrm{M}$ apremilast, the percentage of $\mathrm{G} 0 / \mathrm{G} 1$ phase cells was reduced to $64.1 \%$, compared with the $75.3 \%$ following exposure to IL-17 only. 


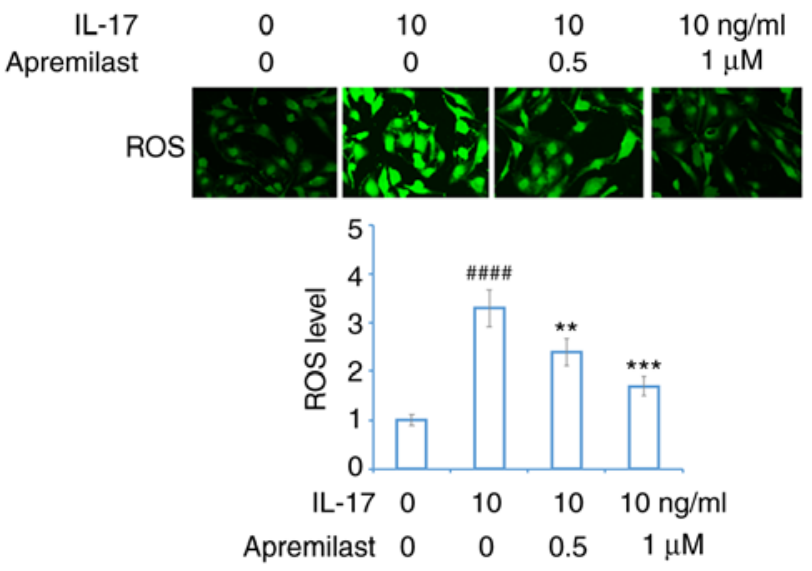

Figure 2. Apremilast prevents IL-17-induced production of ROS in mouse ATDC 5 chondrocytes. Cells were treated with IL-17 $(10 \mathrm{ng} / \mathrm{ml})$ in the presence or absence of apremilast $(0.5$ and $1 \mu \mathrm{M})$ for $24 \mathrm{~h}$. Production of ROS

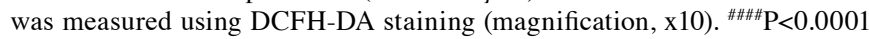
vs. the vehicle control group; ${ }^{* *} \mathrm{P}<0.01$ and ${ }^{* * *} \mathrm{P}<0.001$ vs. the IL-17 group. ROS, reactive oxygen species; IL, interleukin; DCFH-DA, 2,7-dichlorodihydrofluorescein diacetate.
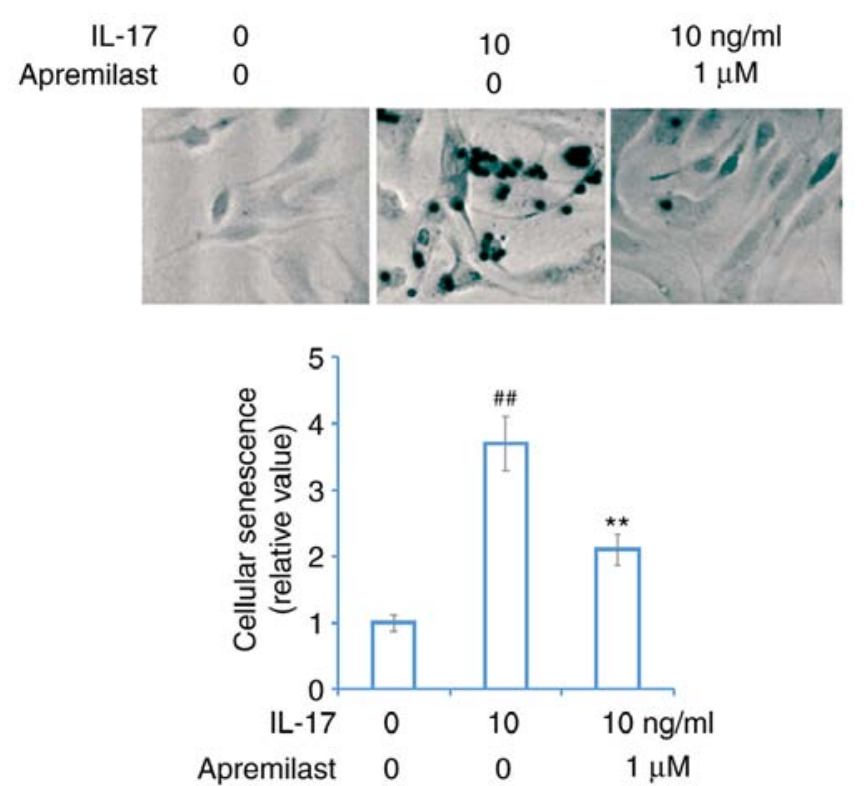

Figure 3. Apremilast prevents IL-17-induced cellular senescence in mouse ATDC5 chondrocytes. Cells were treated with IL-17 $(10 \mathrm{ng} / \mathrm{ml})$ in the presence or absence of apremilast $(1 \mu \mathrm{M})$ for 7 days. Cellular senescence was measured using SA- $\beta$-gal staining. ${ }^{\# \#} \mathrm{P}<0.01$ vs. the vehicle control group; ${ }^{* *} \mathrm{P}<0.01$ vs. the IL-17 group. IL, interleukin; SA- $\beta$-gal, senescence-associa ted- $\beta$-galactosidase.

The percentage of G2/M and S phase cells were rescued to 22.3 and $13.6 \%$, respectively (Fig. 4).

Apremilast inhibits IL-17-induced expression levels of p21 and PAI-1. Thereafter, the effect of apremilast on the expression levels of p21 and PAI-1 were measured. IL-17 stimulation increased the mRNA levels of p21 and PAI-1 to 3.4- and 2.9-fold, respectively, which were reduced to 1.8- and 1.7-fold by $1 \mu \mathrm{M}$ apremilast treatment (Fig. 5A), respectively. Furthermore, apremilast reduced the protein levels of $\mathrm{p} 21$ and PAI-1 to 1.7- and 1.8-fold, respectively, compared to the

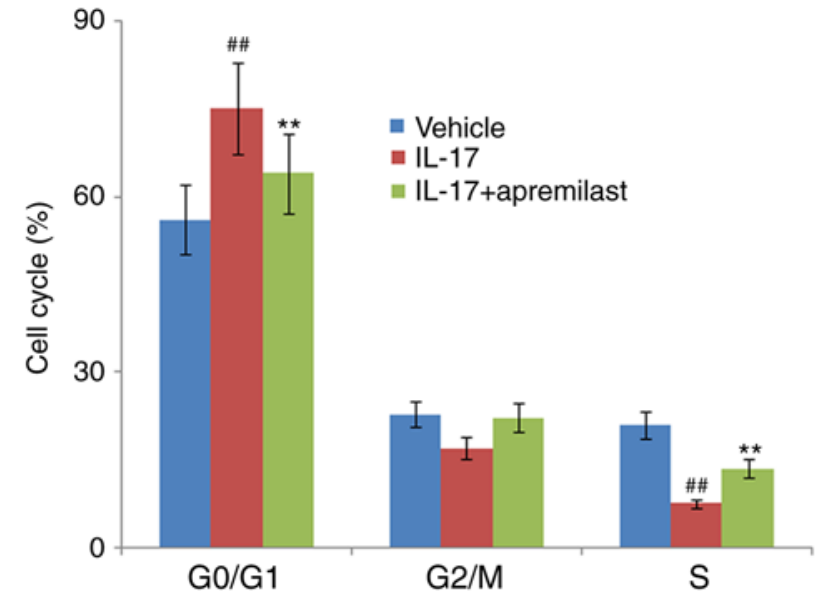

Figure 4. Apremilast prevents IL-17-induced cell cycle arrest in the G0/G1 phase in mouse ATDC5 chondrocytes. Cells were treated with IL-17 $(10 \mathrm{ng} / \mathrm{ml})$ in the presence or absence of apremilast $(1 \mu \mathrm{M})$ for 7 days. The cell cycle was assayed using flow cytometry. G0/G1 phase, G2/M phase, and $\mathrm{S}$ phase of cells were measured. ${ }^{\# \#} \mathrm{P}<0.01$ vs. the vehicle control group; ${ }^{* *} \mathrm{P}<0.01$ vs. the IL-17 group. IL, interleukin.

2.7- and 2.8-fold increase following exposure to IL-17 only (Fig. 5B).

Inhibitory effects of apremilast in cellular senescence are dependent on SIRT1. SIRT1 is one of the most important anti-aging factors (18). In the present study, the influence of apremilast on SIRT1 expression was also assessed. Compared to the non-treated cells, IL-17 reduced the mRNA level of SIRT1 to $53 \%$, which was recovered to $92 \%$, close to the baseline, in the presence of $1 \mu \mathrm{M}$ apremilast (Fig. 6A). It was also confirmed that apremilast rescued the protein level of SIRT1 which had been significantly reduced by IL-17 (Fig. 6B). Moreover, an attempt was made to determine the involvement of SIRT1 in the action of apremilast in chondrocytes. SIRT1 was silenced with SIRT1 siRNA. As revealed in Fig. 7A, in normal SIRT-expressing cells, apremilast significantly suppressed cellular senescence, however, its effect was almost abolished in SIRT1-silenced cells. The results from western blot analysis in Fig. 7B and C revealed that the inhibitory effect of apremilast on PAI-1 and p21 was abolished by the silencing of SIRT1. These data indicated that the effects of apremilast on chondrocytes depend on the expression of SIRT1.

\section{Discussion}

As a common joint degenerative disease, safe and effective therapies against $\mathrm{OA}$ are still required. Aside from inflammation, chondrocyte senescence is considered another important event in the pathobiology of OA (19). Cellular senescence refers to irreversible cell growth stagnation after a characteristic number of cell proliferation (20). One of the most important senescence pathways is called stress-induced premature senescence (SIPS), which can be induced by cytokines, DNA damage, oxidative stress, and external stimuli (21). Excessive production of IL-17 has been linked with chondrocyte activation (22). In the present study, apremilast, a PDE4 inhibitor used for the treatment of psoriasis, was investigated as a potential strategy for slowing chondrocyte senescence in the 

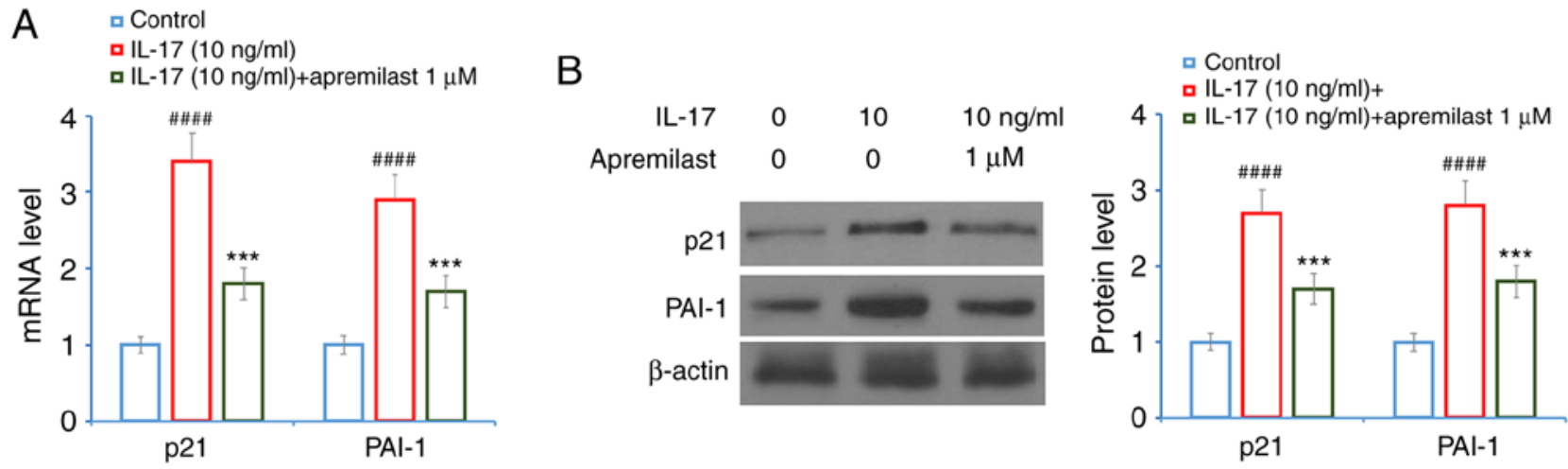

Figure 5. Apremilast prevents IL-17-induced expression of p21 and PAI-1 in mouse ATDC5 chondrocytes. Cells were treated with IL-17 (10 ng/ml) in the presence or absence of apremilast $(1 \mu \mathrm{M})$ for $24 \mathrm{~h}$. (A) mRNA levels of p21 and PAI-1. (B) Protein levels of p21 and PAI-1 as measured by western blotting

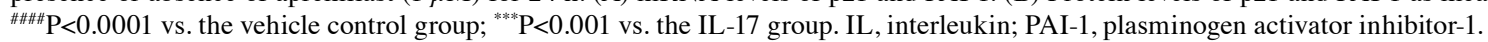
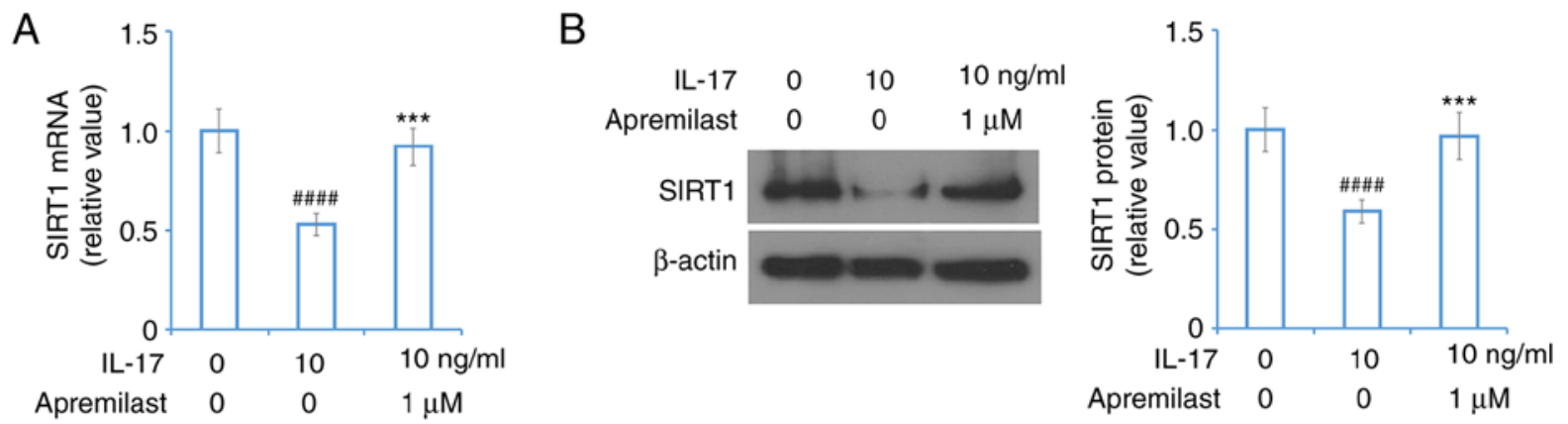

Figure 6. Apremilast prevents IL-17-induced reduction of SIRT1 in mouse ATDC5 chondrocytes. Cells were treated with IL-17 (10 ng/ml) in the presence or absence of a Apremilast $(1 \mu \mathrm{M})$ for $24 \mathrm{~h}$. (A) mRNA of SIRT1. (B) Protein level of SIRT1. ${ }^{\# \# \# "} \mathrm{P}<0.0001$ vs. the vehicle control group; ${ }^{* * *} \mathrm{P}<0.001 \mathrm{vs.}$ the IL-17 group. IL, interleukin; SIRT1, sirtuin 1.

development of OA. First, it was determined that IL-17 treatment significantly increased the production of IL-1 $\beta$, MCP-1, and ROS. IL- $1 \beta$ is one of the most important pro-inflammatory cytokines in various inflammatory diseases including OA, acting as a trigger of the cascades of inflammatory responses. Also, IL-1 $\beta$ has been demonstrated to have a strong effect on chondrocyte senescence (23). MCP-1 also plays a pivotal role in the development of $\mathrm{OA}$ by recruiting immune cells and promoting inflammation (24). Oxidative stress is usually considered as the major inducer of cellular senescence (25). Studies have revealed that increased ROS reduces chondrocyte survival, and this has subsequently been linked to the development of OA (26-28). Further research has demonstrated that ROS could activate several downstream pathways that induce senescence and apoptosis (29).

Furthermore, in this in vitro chondrocyte model, our results indicated the ability of apremilast to inhibit chondrocyte senescence, as revealed by its reduction of senescence-associated $\beta$-galactosidase (SA- $\beta$-gal) activity, which is a cellular marker of senescence (30). Cell cycle arrest is a type of replicative senescence in which cells cease to replicate, and tissue begins to break down. The involvement of expansion and cell cycle arrest in cellular senescence (20). In the present study, it was revealed that IL-17 stimulation elongated quiescent/gap phase (G0/G1), shortened DNA synthesis phase (S phase), and induced senescent phenotype, however, treatment with apremilast reversed the effect of IL-17 on cell cycle arrest.
Mechanistically, it was revealed that apremilast inhibited IL-17-induced expression levels of senescence-related genes. As a downstream gene of p53, the expression of p21 increases in senescent cells and leads to cell cycle arrest by suppressing several cyclin-dependent kinases (31). It has been reported that chondrocyte senescence could be mediated by IL- $1 \beta$ treatment via the $\mathrm{p} 53 / \mathrm{p} 21 / \mathrm{pRb}$ pathway, with the induction of caveolin 1 and activation of $\mathrm{p} 38$ mitogen-activated protein kinase (MAPK), resulting in cell senescence and apoptosis (32). Moreover, researchers have revealed that the expression levels of PAI-1 and p21 proteins have a positive correlation with the presence of SA- $\beta$-gal $(2,33)$. PAI-1 is a member of the evolutionarily conserved serine protease inhibitor (SERPIN) family and is well known as a marker and mediator of senescence, revealed by its increased expression in senescent cells $(34,35)$. Both p21 and PAI-1 are the direct transcriptional targets of p53 (36). The capacity of apremilast to mitigate the expression of p21 and PAI-1 could account for its ameliorative effect on the G1 phase rescue.

Notably, it was revealed that apremilast rescued the expression of SIRT1. SIRT1 is a master metabolic regulator in different metabolic tissues and is associated with various signaling pathways, including inflammation and senescence $(37,38)$. In cartilage tissues, the expression of SIRT1 proteins is present in the nuclei of chondrocytes. Pro-inflammatory cytokines such as TNF- $\alpha$ could inhibit the expression of SIRT1 (39). In the present study, it was revealed that IL-17 also had a strong inhibitory 

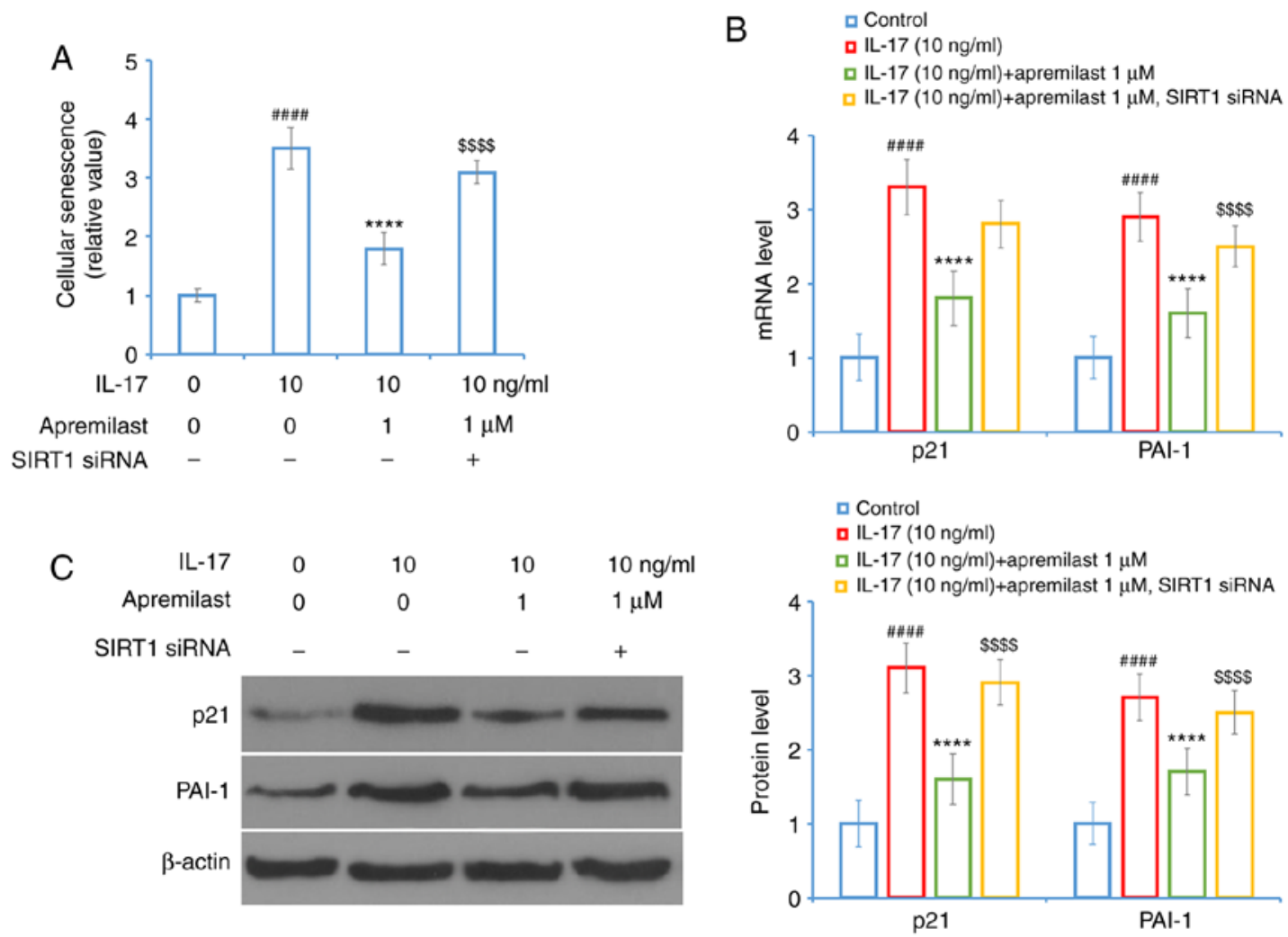

Figure 7. Knockdown of SIRT1 abolishes the protective effects of apremilast against IL-17-induced cellular senescence and the expression levels of p21 and PAI-1. Cells were transfected with SIRT1 siRNA, followed by stimulation with IL-17 $(10 \mathrm{ng} / \mathrm{ml})$ in the presence or absence of apremilast $(1 \mu \mathrm{M})$ for $24 \mathrm{~h}$. (A) Cellular senescence. (B) mRNA levels of p21 and PAI-1. (C) Protein levels of p21 and PAI-1 as measured by western blotting. \#\#\#\# P<0.0001 vs. the vehicle control group; ${ }^{* * * * *} \mathrm{P}<0.0001$ vs. the IL-17 group; ${ }^{\$ \$ \$ \$} \mathrm{P}<0.0001$ vs. the IL-17 + apremilast group. SIRT1, sirtuin 1; IL, interleukin; PAI-1, plasminogen activator inhibitor-1.

effect on the expression of SIRT1. It has been demonstrated that the expression of SIRT1 is decreased in degenerated cartilage, resulting in inflammation and chondrocyte senescence (40). However, the function of SIRT1 in OA has not been entirely elucidated. Recently, studies revealed evidence that SIRT1 regulates the expression of extracellular matrix proteins, and silencing of SIRT1 in chondrocytes leads to osteoarthritic gene expression $(40,41)$. Based on this, SIRT1 was considered as a key regulator in the prevention of chondrocyte senescence by apremilast. As anticipated, apremilast rescued the expression of SIRT1 reduced by IL-17. In addition, silencing of SIRT1 abolished the protective effects of apremilast against IL-17induced cellular senescence. A graphical representation of the underlying mechanism is presented in Fig. 8.

Monoclonal antibodies mediating IL-17 inhibition have been used in the treatment of rheumatoid arthritis (RA), with conflicting results having been reported $(9,42)$. However, IL-17 inhibition appears to be effective in the treatment of psoriasis (43). Notably, a recent study revealed that intra-articular injection of an IL-17-neutralizing antibody reduced joint degeneration in aging OA mice (44). However, IL-17 inhibition has not been trialed in the treatment of OA in patients. Given the heterogeneity of RA and OA, the inhibition of IL-17 may not produce the same outcome in patients. More preclinical data are required for the implementation of the IL-17 inhibition approach in human OA.

Collectively, the present findings revealed that apremilast possessed a protective capacity against IL-17-induced cellular senescence in chondrocytes. Further investigations are

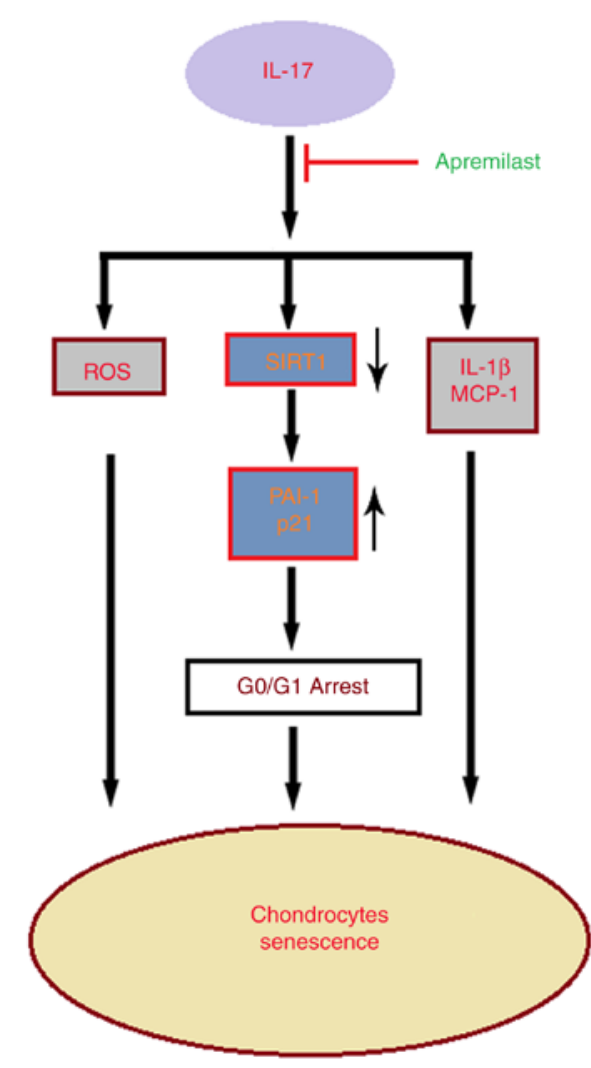

Figure 8. Graphical representation of the underlying mechanism, whereby apremilast prevents IL-17-induced cellular senescence in ATDC5 chondrocytes. IL, interleukin; ROS, reactive oxygen species; SIRT1, sirtuin 1; MCP-1, monocyte chemoattractant protein-1; PAI-1, plasminogen activator inhibitor-1. 
required to clarify the mechanisms driving these protective effects of apremilast.

\section{Acknowledgements}

Not applicable.

\section{Funding}

No funding was received.

\section{Availability of data and materials}

The datasets used during the present study are available from the corresponding author upon reasonable request.

\section{Authors' contributions}

BW and FL contributed to the conception and design of the study. BW, WS, KB and YL contributed to the acquisition of the data. BW and FL contributed to the analysis of the data. FL contributed to the drafting of the manuscript. All authors read and approved the manuscript and agree to be accountable for all aspects of the research in ensuring that the accuracy or integrity of any part of the work are appropriately investigated and resolved.

\section{Ethics approval and consent to participate}

Not applicable.

\section{Patient consent for publication}

Not applicable.

\section{Competing interests}

The authors declare that they have no competing interests.

\section{References}

1. McCulloch K, Litherland GJ and Rai TS: Cellular senescence in osteoarthritis pathology. Aging Cell 16: 210-218, 2017.

2. Loeser RF: Aging and osteoarthritis: The role of chondrocyte senescence and aging changes in the cartilage matrix. Osteoarthritis Cartilage 17: 971-979, 2009.

3. Singh P, Marcu KB, Goldring MB and Otero M: Phenotypic instability of chondrocytes in osteoarthritis: On a path to hypertrophy. Ann N Y Acad Sci 1442: 17-34, 2019.

4. Greene MA and Loeser RF: Aging-related inflammation in osteoarthritis. Osteoarthritis Cartilage 23: 1966-1971, 2015.

5. Liu T, Yang Q, Zhang X, Qin R, Shan W, Zhang H and Chen X: Quercetin alleviates kidney fibrosis by reducing renal tubular epithelial cell senescence through the SIRT1/PINK1/mitophagy axis. Life Sci 257: 118116, 2020.

6. Schön MP: Adaptive and innate immunity in psoriasis and other inflammatory disorders. Front Immunol 10: 1764, 2019.

7. Neogi $T$ and Zhang Y: Epidemiology of osteoarthritis. Rheum Dis Clin North Am 39: 1-19, 2013.

8. Agarwal S, Misra R and Aggarwal A: Interleukin 17 levels are increased in juvenile idiopathic arthritis synovial fluid and induce synovial fibroblasts to produce proinflammatory cytokines and matrix metalloproteinases. J Rheumatol 35: 515-519, 2008.

9. Robert M and Miossec P: IL-17 in rheumatoid arthritis and precision medicine: From synovitis expression to circulating bioactive levels. Front Med (Lausanne) 5: 364, 2019.
10. Wojdasiewicz P, Poniatowski ŁA and Szukiewicz D: The role of inflammatory and anti-inflammatory cytokines in the pathogenesis of osteoarthritis. Mediators Inflamm 2014: 561459, 2014

11. Honorati MC, Bovara M, Cattini L, Piacentini A and Facchini A: Contribution of interleukin 17 to human cartilage degradation and synovial inflammation in osteoarthritis. Osteoarthritis Cartilage 10: 799-807, 2002.

12. Lee YH and Bae SC: Associations between circulating IL-17 levels and rheumatoid arthritis and between IL-17 gene polymorphisms and disease susceptibility: A meta-analysis. Postgrad Med J 93: 465-471, 2017.

13. Otezla ${ }^{\circledR}$ (package insert). Celgene Corporation, Summit, NJ, 2014.

14. Lanna C, Cesaroni GM, Mazzilli S, Vollono L, Gaziano R, Marino D, Bianchi L and Campione E: Apremilast as a target therapy for nail psoriasis: A real-life observational study proving its efficacy in restoring the nail unit. J Dermatolog Treat: Aug 3, 2020 (Epub ahead of print).

15. Schafer PH, Adams M, Horan G, Truzzi F, Marconi A and Pincelli C: Apremilast normalizes gene expression of inflammatory mediators in human keratinocytes and reduces Antigen-Induced Atopic Dermatitis in mice. Drugs R D 19: 329-338, 2019.

16. Mavropoulos A, Zafiriou E, Simopoulou T, Brotis AG, Liaskos C, Roussaki-Schulze A, Katsiari CG, Bogdanos DP and Sakkas LI: Apremilast increases IL-10-producing regulatory B cells and decreases proinflammatory $\mathrm{T}$ cells and innate cells in psoriatic arthritis and psoriasis. Rheumatology (Oxford) 58: 2240-2250, 2019.

17. Livak KJ and Schmittgen TD: Analysis of relative gene expression data using real-time quantitative PCR and the 2(-Delta Delta C(T)) method. Methods 25: 402-408, 2001.

18. Sarubbo F, Esteban S, Miralles A and Moranta D: Effects of resveratrol and other polyphenols on Sirt1: Relevance to brain function during aging. Curr Neuropharmacol 16: 126-136, 2018.

19. Hwang HS and Kim HA: Chondrocyte apoptosis in the pathogenesis of osteoarthritis. Int J Mol Sci 16: 26035-26054, 2015.

20. Toh WS, Brittberg M, Farr J, Foldager CB, Gomoll AH, Hui JH, Richardson JB, Roberts S and Spector M: Cellular senescence in aging and osteoarthritis. Acta Orthop 87 (Sup363): 6-14, 2016.

21. Musumeci G, Szychlinska MA and Mobasheri A: Age-related degeneration of articular cartilage in the pathogenesis of osteoarthritis: Molecular markers of senescent chondrocytes. Histol Histopathol 30: 1-12, 2015.

22. Malemud CJ: Cytokines as therapeutic targets for osteoarthritis. BioDrugs 18: 23-35, 2004.

23. Wang M, Liu L, Zhang CS, Liao Z, Jing X, Fishers M, Zhao L, $\mathrm{Xu} \mathrm{X}$ and Li B: Mechanism of traditional Chinese medicine in treating knee osteoarthritis. J Pain Res 13: 1421-1429, 2020.

24. Miller RE, Miller RJ and Malfait AM: Osteoarthritis joint pain: The cytokine connection. Cytokine 70: 185-193, 2014.

25. Ashraf S, Cha BH, Kim JS, Ahn J, Han I, Park H and Lee SH: Regulation of senescence associated signaling mechanisms in chondrocytes for cartilage tissue regeneration. Osteoarthritis Cartilage 24: 196-205, 2016.

26. Lepetsos $P$ and Papavassiliou AG: ROS/oxidative stress signaling in osteoarthritis. Biochim Biophys Acta 1862: 576-591, 2016.

27. Drevet S, Gavazzi G, Grange L, Dupuy C and Lardy B: Reactive oxygen species and NADPH oxidase 4 involvement in osteoarthritis. Exp Gerontol 111: 107-117, 2018.

28. Zahan OM, Serban O, Gherman C and Fodor D: The evaluation of oxidative stress in osteoarthritis. Med Pharm Rep 93: 12-22, 2020.

29. Li YS, Xiao WF and Luo W: Cellular aging towards osteoarthritis. Mech Ageing Dev 162: 80-84, 2017.

30. Takács-Buia L, Iordachel C, Efimov N, Caloianu M, Montreuil J and Bratosin D: Pathogenesis of osteoarthritis: Chondrocyte replicative senescence or apoptosis? Cytometry B Clin Cytom 74: 356-362, 2008

31. Regulski MJ: Cellular Senescence: What, why, and how. Wounds 29: 168-174, 2017.

32. Dai SM, Shan ZZ, Nakamura H, Masuko-Hongo K, Kato T, Nishioka K and Yudoh K: Catabolic stress induces features of chondrocyte senescence through overexpression of caveolin 1: Possible involvement of caveolin 1-induced down-regulation of articular chondrocytes in the pathogenesis of osteoarthritis. Arthritis Rheum 54: 818-831, 2006.

33. Guo Y, Fan W, Xie Y, Cao S, Wan H and Jin B: SIRT1 is the target gene for $2,3,5,4$ '-tetrahydroxystilbene-2-O- $\beta$-D-Gluco side alleviating the HUVEC senescence. Front Pharmacol 11: 542902, 2020. 
34. Vaughan DE, Rai R, Khan SS, Eren $M$ and Ghosh AK: Plasminogen activator inhibitor-1 is a marker and a mediator of senescence. Arterioscler Thromb Vasc Biol 37: 1446-1452, 2017.

35. Kortlever RM, Higgins PJ and Bernards R: Plasminogen activator inhibitor-1 is a critical downstream target of p53 in the induction of replicative senescence. Nat Cell Biol 8: 877-884, 2006.

36. Samarakoon R, Higgins SP, Higgins CE and Higgins PJ: The TGF- $\beta 1 /$ p53/PAI-1 signaling axis in vascular senescence: Role of caveolin-1. Biomolecules 9: 341, 2019.

37. Li X: SIRT1 and energy metabolism. Acta Biochim Biophys Sin (Shanghai) 45: 51-60, 2013

38. Takayama K, Ishida K, Matsushita T, Fujita N, Hayashi S, Sasaki K, Tei K, Kubo S, Matsumoto T, Fujioka H, et al: SIRT1 regulation of apoptosis of human chondrocytes. Arthritis Rheum 60: 2731-2740, 2009.

39. Dvir-Ginzberg M, Gagarina V, Lee EJ, Booth R, Gabay O and Hall DJ: Tumor necrosis factor $\alpha$-mediated cleavage and inactivation of SirT1 in human osteoarthritic chondrocytes. Arthritis Rheum 63: 2363-2373, 2011.

40. Fujita N, Matsushita T, Ishida K, Kubo S, Matsumoto T, Takayama K, Kurosaka M and Kuroda R: Potential involvement of SIRT1 in the pathogenesis of osteoarthritis through the modulation of chondrocyte gene expressions. J Orthop Res 29: 511-515, 2011.
41. Gabay O, Zaal KJ, Sanchez C, Dvir-Ginzberg M, Gagarina V, Song Y, He XH and McBurney MW: Sirt1-deficient mice exhibit an altered cartilage phenotype. Joint Bone Spine 80: 613-620, 2013.

42. Wei M and Duan D: Efficacy and safety of monoclonal antibodies targeting interleukin-17 pathway for inflammatory arthritis: A meta-analysis of randomized controlled clinical trials. Drug Des Devel Ther 10: 2771-2777, 2016.

43. Miossec P: Update on interleukin-17: A role in the pathogenesis of inflammatory arthritis and implication for clinical practice. RMD Open 3: e000284, 2017.

44. Faust HJ, Zhang H, Han J, Wolf MT, Jeon OH, Sadtler K, Peña AN, Chung L, Maestas DR Jr, Tam AJ, et al: IL-17 and immunologically induced senescence regulate response to injury in osteoarthritis. J Clin Invest 130: 5493-5507, 2020.

This work is licensed under a Creative Commons Attribution-NonCommercial-NoDerivatives 4.0 International (CC BY-NC-ND 4.0) License. 